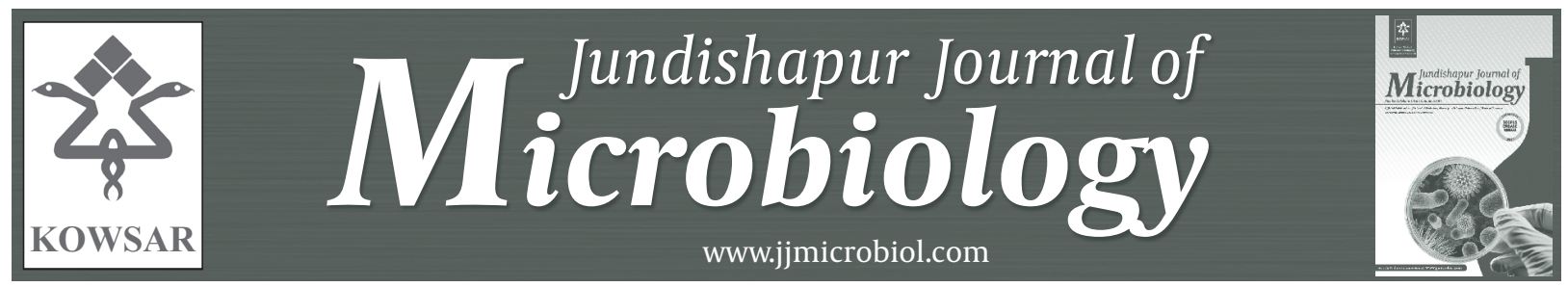

\title{
The Prevalence of Active Tuberculosis Among Patients With Fibrotic Lesion in Chest CT-Scan
}

\author{
Seyed Ali Javad Moosavi ${ }^{1}$, Hanieh Raji ${ }^{2,}$, , Mahshid Talebi-Taher ${ }^{3}$, Shadi Ghourchian ${ }^{4}$ \\ ${ }^{1}$ Internal Medicine Department, Tehran University of Medical Sciences, Tehran, IR Iran \\ ${ }^{2}$ Internal Medicine Department, Jundishapur University of Medical Sciences and Health Services, Ahvaz, IR Iran \\ ${ }^{3}$ Internal Medicine Department, Tehran University of Medical Sciences, Tehran, IR Iran \\ ${ }^{4}$ Scientific Research Center, Tehran University of Medical Sciences, Tehran, IR Iran \\ *Corresponding author: Hanieh Raji, Jundishapur University of Medical Sciences and Health Services, Ahvaz, IR Iran. Tel: +98-6114163728, E-mail: dr.raji.h@ \\ gmail.com.
}

\begin{abstract}
A B S T R A C T
Background: Since tuberculosis (TB) is a major public health problem that is a leading cause of mortality and morbidity among infectious diseases worldwide, early diagnosis and treatment are important to control an effective tuberculosis (TB) and also the increasing number of patients with atypical manifestations of active TB. It suggests more evaluation for active TB in fibrotic lesion in CT scan.

Objectives: We evaluated patients with each respiratory complaints and apical fibrocalcification in chest CT scan to detect active TB.

Patients and Methods: This study was an observational cross sectional study and was carried out from July 2010 to September 2011 in our teaching hospital. Patients with apical fibrocalcification or fibrocystic lesion in lung CT scan (regardless of the size), without history of TB or other diseases which can cause these lesions were enrolled, then sputum analysis was performed, and in case the result was negative, we did bronchoalveolar lavage for them.

Results: We gathered 40 patients out of which 15 patients were women. The average age was calculated at $64 \pm 8$ years old. In total 6 patients had positive results.

Conclusions: According to our observations fibrocalcified lesions should be evaluated for detecting M. tuberculosis particularly in the endemic regions.
\end{abstract}

Keywords: Mycobacterium tuberculosis; Pulmonary tuberculosis; Pulmonary fibrosis; Spiral CT Scan

Copyright $\odot$ 2013, Ahvaz Jundishapur University of Medical Sciences; Published by Kowsar Corp.

Article type: Research Article; Received: 09 May 2012; Revised: 23 Jul 2012; Accepted: 06 Aug 2012; Epub: 01 Jun 2013; Ppub: Jun 2013 Implication for health policy/practice/research/medical education:

Pulmonary tuberculosis is a serious infection, therefore, delay in diagnosis and treatment can cause morbidity and mortality. Considering epidemiological aspects and also the increasing number of patients with atypical manifestations of active TB compared to common symptoms, the importance of finding new cases involved in active TB deserves more investigations especially in the areas with high prevalence of TB.

Please cite this paper as:

Moosavi SAJ, Raji H, Talebi Taher M, Ghourchian S. The Prevalence of Active Tuberculosis Among Patients With Fibrotic Lesion in Chest CT-Scan. Jundishapur J Microbiol. 2013;6(4):e5179. DOI:10.5812/jjm.5179.

Copyright @ 2013, Ahvaz Jundishapur University of Medical Sciences; Published by Kowsar Corp.

This is an Open Access article distributed under the terms of the Creative Commons Attribution License (http://creativecommons.org/licenses/by/3.0), which permits unrestricted use, distribution, and reproduction in any medium, provided the original work is properly cited. 


\section{Background}

Tuberculosis (TB) is a major public health problem that is a leading cause of mortality and morbidity among infectious diseases worldwide. Early diagnosis and treatment are essential for an effective tuberculosis (TB) control program (1). For both disease prognosis at the individual level and transmission within the community, delay in diagnosis is important. Most transmissions occur between the onset of cough and initiation of treatment (2).

Lungs are the most common sites involved in Mycobacterium tuberculosis. Radiological findings may be very diverse. Radiological equipments including Chest-X-Ray (CXR) and CT scan are used to help diagnosis by showing some exclusive findings for TB, however, they cannot replace bacteriological confirmation of the diagnosis (3-8). CT-scan seems to be more sensitive than CXR in showing smaller lesions. Although the lesions "cavitation and tree in bud" are strongly suggestive of active TB specially in symptomatic patients but the active $M$. tuberculosis has been found in fibrocalcified lesions of lungs(6, 8-13).

M.tuberculosis usually exists in the upper parts of the lung due to higher concentration of oxygen and the absence of suitable lymphatic drainage in these parts comparing with other segments (5). It causes higher resistance rate and virulence of bacilli in upper parts, but the existence of active bacilli in fibrocalcification lesions surrounded apical parts of lung is not found in previous studies according to our knowledge.

\section{Objectives}

Considering epidemiological aspects and also the increasing number of patients with atypical manifestations of active TB compared to common symptoms, the importance of finding new cases involved by active TB deserves more investigations especially in Iran which is considered as one of the countries with a high prevalence of TB. We aimed at detecting M. tuberculosis in patients with fibrocalcified lesions in apical parts of the lungs regardless of the size of lesions.

\section{Patients and Methods}

This observational cross sectional study was supported by the institutional review board of Tehran University of Medical Sciences and, it was carried out from July 2010 to September 2011 in our teaching hospitals. All patients were convinced by the authors that their CT-scan findings will be entered as data in this study and they will be evaluated for active TB. Informed consent was obtained.

Patients who were referred to the internal ward and pulmonary clinic of hospital with respiratory complaints were undergone CT-scan. We included patients with fibrocalcification (Figure 1) and fibrocystic lesion in the apical lungs regardless of its size in CT- scan and without any former radiographic for comparison, with those who had no history of TB or other diseases which could have caused such lesions, were enrolled.

After gathering 40 patients with uttered inclusion criteria they underwent physical examination to confirm that they did not have any findings compatible with active TB, and then sputum analysis (smear and standard culture for bacteriological confirmation) was performed for all patients, and when the result was negative, we did bronchoalveolar lavage for them.

We aimed at detecting detect M. tuberculosis in patients with fibrocalcified lesions in apical parts of their lungs regardless of the size of lesions.

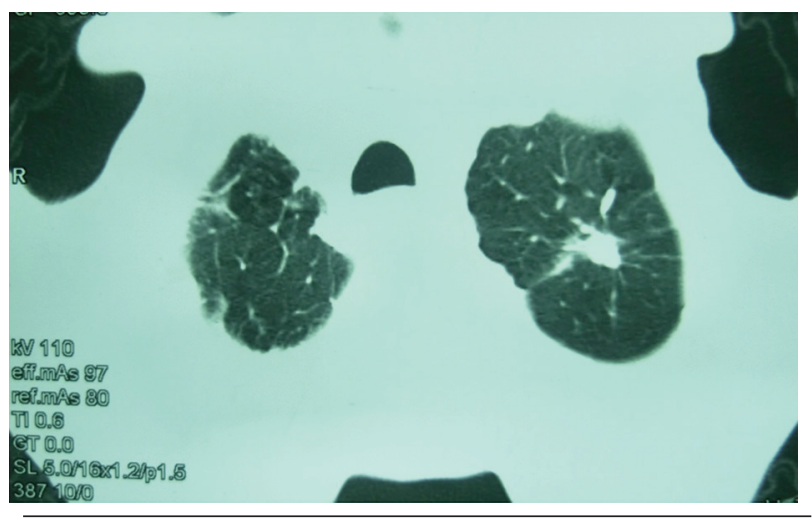

Figure 1. Fibrotic Lesion in Left Upper Lobe

\section{Results}

From 40 patients who were evaluated, 15 patients were women. The average age was calculated at $64 \pm 8$. All of the cases had normal bronchoscopy except 3 patients who had anthracosis. The numbers of involvement in the apex of the left lung, the right lung and in both apical segments of lungs in CT scan were 7, 13 and 20 respectively. Beyond CT-scan findings, the confirming test revealed the following results. One patient had positive smear and culture of sputum for M. tuberculosis, and the smear and culture of lavage appeared positive in two patients and just positive culture of lavage was shown in 3 patients (smear negative culture positive). In total 6 patients showed positive results.

\section{Discussion}

Up to this time, one third of the population of the world has been infected by tuberculosis. The incidence rate of TB is estimated more than 9 million people per year from which them 500,000 are drug resistant $(4,14,15)$. Typical symptoms include low grade fever, productive cough (usually longer than 2 weeks, thin, odorless, and yellowish color), chest pain, night sweats, varying degrees of hemoptysis in more than $25 \%$ of the patients and in later stages, weight loss, progressive debilitation, and cachex- 
ia $(2,6,15-17)$.

Active TB usually appears in patients with predisposing factors such as malnutrition, cancer or diabetes (1517). CXR is the most important screening tool. Among all radiological presentations, cavitation, which is seen in more than half of the patients, is the hallmark for TB and can be presented in different locations and triplicates the probability of TB recurrence $(15,18)$. Previous studies revealed that scarring and nodules following primary TB are supposed to be gotten after healing. Also some studies showed that Tuberculomas which are described as well-defined nodules located in upper parts of the lung and calcification in $20-30 \%$ of cases may appear after primary TB or recurrence of $M$. tuberculosis. Radiological findings are the most assistant way for following these patients (19).

Although CT scan provides better discrimination of nodules and calcifications and can present some masses which are not visible on CXR, the diagnosis should be confirmed by scrutinizing of sputum $(6,8,19,20)$. Fibrotic mass like lesions in CT-scan without any symptoms usually are not accounted for active TB and so are ignored in most patients, but in our study we found patients without any previous or present TB symptoms who carried bacilli Koch in endemic areas where drug resistance is increasing.

Though lower lung zones involvement is more predominant in primary $\mathrm{TB}$, patchy, poorly defined infiltrates or consolidated areas in the apical and posterior upper lobes, and less frequently, the superior segments of the lower lobes, with or without cavitation are supposed pictures in post primary TB. Between the two, involvement of the left side is more often destructive $(6,13,18,21)$.

Sant'Anna et al. in a retrospective, cross-sectional, observational study of 850 patients with TB reported the most common radiologic lesion by CXR was the upper pulmonary lobe infiltrate (53.3\%). Isolated cavitation was found in $32.4 \%$ of the patients, both lungs were affected in $29.2 \%$ of the patients, normal CXR in $6.2 \%(22)$. According to this study, TB can present in normal CXR, but for patients with normal CXR, CT scan is not performed. This is while CT scan could show some lesions that are not visible on CXR such as small granuloma and fibrotic lesion.

Jamal et al. evaluated frequency of atypical radiological pattern of pulmonary tuberculosis in adults and elderly, in their study typical radiological pattern means infiltration and / or nodules with or without cavitations, involving upper zone while the pattern other than typical one is assumed as atypical. Out of 300 patients, infiltration was observed in 141 (47.0\%), nodule in 99 (33.0\%), and cavitation in 60 (20.0\%) patients. Study revealed that atypical radiographic appearances were more common in elderly group. Young adult patients presented with classical symptoms of TB, and also had greater frequency of upper zone infiltration on chest radiograph (23).

In 2003 Jin Mo Goo and colleagues investigated the prevalence of active TB in 143 patients with idiopathic pulmonary fibrosis (IPF). Among them the overall prevalence of tuberculosis was 6.29\% (9/143). Mass like lesion was the most prevalent shape found in CT-scan in that study (6 patients). Segmental or lobar consolidation was another finding in CT-scan in the rest of patients (3 patients). From those three patients two of them had large necrotizing cavities in the area of consolidation. Through their findings, typical patterns of tuberculosis (multifocal patchy consolidations or centrilobular nodules) were found to be the least (2/9). Also right lower lobe was the predominant segment for showing the lesions (24). There was no finding described as an apical fibrocalcification in patients with active TB.

In 2010 , as the last research about radiological findings in TB in our literature review, Eisenberg Rl and et al, evaluated 2585 persons with the purpose of assessing "the frequency and spectrum of abnormalities on routine screening chest radiographs in the pre-employment evaluation of health care workers with positive tuberculin skin test (TST) results". Although there were 108 cases with calcification or fibrosis out of the total 159 with abnormal findings in CXR, they didn't find any criteria for active TB in radiology. As they mentioned they didn't try for acid fast bacilli in calcified or fibrotic lesions, though they tried it in noncalcified nodules (25).

Our study is the first evaluation for active bacilli in fibrotic lesions in upper part of lung and one of limitations for this study is low sample size. By our observation fibrocalcified lesions should be evaluated for active TB specifically in this era which the prevalence of patients with active TB presented with atypical manifestations is increasing (24). Besides, patients with these manifestations are ignored even though they are a reservoir for distributing M. tuberculosis (25). So we suggest that not only the patients with unexplained productive coughs for more than 2-3 weeks, but also patients with other symptoms or even without symptoms or typical radiological findings, but with apical fibrocalcification would be be evaluated for active TB by sputum analysis and bronchoalveolar lavage if the result of sputum was negative, especially in endemic areas such as our country.

\section{Acknowledgements}

We are grateful to Dr Nader Afshari resident of cardiology Ahvaz Jundishapur University of Medical Sciences for editing.

\section{Financial Disclosure}

Non declared.

\section{Funding/Support}

None declared. 


\section{Authors' Contribution}

None declared.

\section{References}

1. Bento J, Silva AS, Rodrigues F, Duarte R. [Métodos Diagnósticos Em Tuberculose]. Acta Med Port. 2011;24:145-154.

2. Storla DG, Yimer S, Bjune G. A systematic review of delay in the diagnosis and treatment of tuberculosis. BMC Public Health. 2008;8(1):15.

3. Vinh DC, Menzies D. Sputum induction for diagnosis of pulmonary tuberculosis: ready for prime time? Chest. 2006;130(5):16267.

4. Small PM, Fujiwara PI. Management of tuberculosis in the United States. N Engl J Med. 2001;345(3):189-200.

5. McAdams HP, Erasmus J, Winter JA. Radiologic manifestations of pulmonary tuberculosis. Radiol Clin North Am. 1995;33(4):655-78.

6. Jeong YJ, Lee KS. Pulmonary tuberculosis: up-to-date imaging and management. AJR Am J Roentgenol. 2008;191(3):834-44.

7. Mor Z, Leventhal A, Weiler-Ravell D, Peled N, Lerman Y. Chest Radiography Validity in Screening Pulmonary Tuberculosis in Immigrants From a High-Burden Country. Respir Care. 2012;57(7):113744.

8. Wormanns D. [Radiological imaging of pulmonary tuberculosis]. Radiologe. 2012;52(2):173.

9. Akira M, Sakatani M. Clinical and high-resolution computed tomographic findings in five patients with pulmonary tuberculosis who developed respiratory failure following chemotherapy. Clin Radiol. 2001;56(7):550-5.

10. Burrill J, Williams CJ, Bain G, Conder G, Hine AL, Misra RR. Tuberculosis: A Radiologic Review1. Radiographics. 2007;27(5):1255-1273.

11. Hatipoglu ON, Osma E, Manisali M, Ucan ES, Balci P, Akkoclu A, et al. High resolution computed tomographic findings in pulmonary tuberculosis. Thorax. 1996;51(4):397-402.

12. Im JG, Webb WR, Han MC, Park JH. Apical opacity associated with pulmonary tuberculosis: high-resolution CT findings. Radiology. 1991;178(3):727-31.

13. Poey C, Verhaegen F, Giron J, Lavayssiere J, Fajadet P, Duparc B. High resolution chest CT in tuberculosis: evolutive patterns and signs of activity.J Comput Assist Tomogr.1997;21(4):601-7.

14. Hopewell PC. A clinical view of tuberculosis. Radiol Clin North Am 1995;33(4):641-53.

15. Quast TM, Browning RF. Pathogenesis and clinical manifestations of pulmonary tuberculosis. Dis Mon. 2006;52(11-12):413-9.

16. Neralla S, Glassroth J. In: Shields T.W, editor(s). Pulmonary tuberculosis and other mycobacterial diseases of the lung. Philadelphia : Lippincott Williams \& Wilkins,; 2005.

17. Sant'Anna C, March MF, Barreto M, Pereira S, Schmidt C. Pulmonary tuberculosis in adolescents: radiographic features. Int J Tuberc Lung Dis. 2009;13(12):1566-8.

18. Andreu J, Caceres J, Pallisa E, Martinez-Rodriguez M. Radiological manifestations of pulmonary tuberculosis. Eur J Radiol. 2004;51(2):139-49.

19. Miller WT, Miller WT, Jr. Tuberculosis in the normal host: radiological findings. Semin Roentgenol.1993;28(2):109-18.

20. Kim WS, Moon WK, Kim IO, Lee HJ, Im JG, Yeon KM, et al. Pulmonary tuberculosis in children: evaluation with CT. AJR Am J Roentgenol.1997;168(4):1005-9.

21. Goo JM, Im JG. CT of tuberculosis and nontuberculous mycobacterial infections. Radiol Clin North Am. 2002;40(1):73-87.

22. Sant'Anna CC, Schmidt CM, March MFBP, Pereira SM, Barreto ML. Radiologic findings of pulmonary tuberculosis in adolescents. Brazil J Infect Dis. 2011;15(1):40-44.

23. Jamal AN, Pasha MB, Pasha AK, Raza AA, Pasha MB. Frequency of Atypical Radiological Pattern of Pulmonary Tuberculosis in Adults and Elderly (Dissertation Based Article). Annals of KEMU. 2011;17(2)

24. Chung MJ, Goo JM, Im JG. Pulmonary tuberculosis in patients with idiopathic pulmonary fibrosis. EurJ Radiol. 2004;52(2):175-9.

25. Eisenberg RL, Pollock NR. Low yield of chest radiography in a large tuberculosis screening program. Radiology. 2010;256(3):998-1004. 